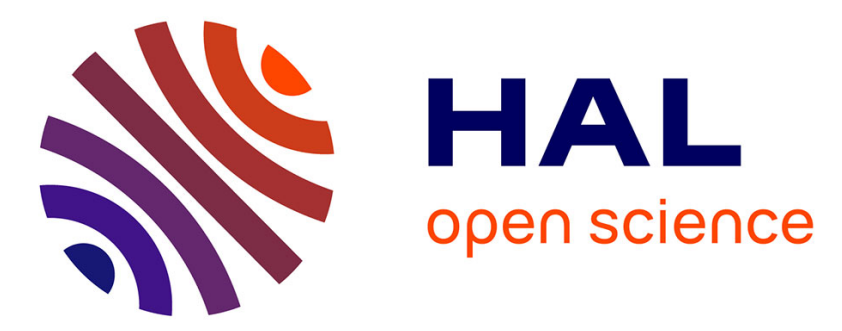

\title{
Acoustic noise emitted by passive components in magnetic devices and design of a low-noise industrial inductor
}

Olivier Barre, Napame Bellemain, Michel Hecquet, Pascal Brochet

\section{- To cite this version:}

Olivier Barre, Napame Bellemain, Michel Hecquet, Pascal Brochet. Acoustic noise emitted by passive components in magnetic devices and design of a low-noise industrial inductor. COMPEL: The International Journal for Computation and Mathematics in Electrical and Electronic Engineering, 2008. hal-01730151

\author{
HAL Id: hal-01730151 \\ https://hal.science/hal-01730151
}

Submitted on 13 Mar 2018

HAL is a multi-disciplinary open access archive for the deposit and dissemination of scientific research documents, whether they are published or not. The documents may come from teaching and research institutions in France or abroad, or from public or private research centers.
L'archive ouverte pluridisciplinaire HAL, est destinée au dépôt et à la diffusion de documents scientifiques de niveau recherche, publiés ou non, émanant des établissements d'enseignement et de recherche français ou étrangers, des laboratoires publics ou privés. 


\title{
Acoustic noise emitted by passive components in magnetic devices and design of a low-noise industrial inductor
}

\author{
Olivier BARRE, Napame BELLEMAIN, Michel HECQUET and Pascal BROCHET \\ L2EP - ECOLE CENTRALE DE LILLE, Villeneuve d'Ascq (France)
}

\author{
Olivier.barre@ec-lille.fr - bellemainn@yahoo.fr - michel.hecquet@ec-lille.fr - pascal.brochet@ec-lille.fr
}

\begin{abstract}
Purpose - The purpose of this paper is to present the original study of an industrial device. Industrial inductors are used to decrease the current variations, resulting from the use of modern power converters. To reduce these variations, the magnetic energy stored in these components is automatically used when the receptor is unconnected to the principal sources. Such storage is generally obtained by using a magnetic circuit containing air-gaps. The rigidity of this circuit, associated to the magnetic stresses which appear in these areas leads the structure to produce mechanical vibration and to emit audible sounds. Design/methodology/approach - Experiments, simulations and test devices are used to determine the main physical phenomena that generates the undesirable audible noise. The resulting knowledge is used to design a quieter device.

Findings - The mechanical vibrations and emitted noises are attached to magnetic effects. Even if it is not possible to suppress all these effects, the level of sound emitted can be decreased through a suitable design of the magnetic core.

Originality/value - Industrial inductors are usually built and designed using methods coming from the transformer studies. A new concept for the design of the magnetic core is presented. Experimental approaches and numerical simulations are performed in order to point out the physical behaviours of the coils and their magnetic coupling to the magnetic core. It appears that breaking the magnetic core into free parts is an original solution that decreases the emitted noise.
\end{abstract}

Keywords - numerical resolutions, mechanical stress, magnetic field effects, power apparatus design, acoustic noise.

Paper type - Research paper

\section{Introduction.}

Electromagnetic devices; such as transformers and the electric motors, have been used for a century. Their acoustic behaviours have been and are still studied. The acoustic behaviour of an electric motor is different from the acoustic behaviour of a transformer. The inductors are commonly used in electronic power systems. The electronic switches introduce a high efficiency in terms of power conversion but induce current modulations. These modulations generate undesirable noise. The magnetic core is often presented as the main source of noise in magnetic devices such as transformers or inductors, but this element is not a source of magnetic field. It only undergoes a mechanical excitation coming from the magnetic field and this one is imposed by the coils. The mechanical behaviour of the entire magnetic core is often studied. Magnetostriction, local mechanical vibrations of the magnetic sheets constituting the magnetic core and Lorentz-forces are the physical phenomena associated to this device that are usually investigated. This article first studies the effect of coils. They are the only active parts in the noise emissions and their study can be a source of interesting information to produce solutions to reduce noise, even if the magnetic core is usually considered as the main domain of investigation. This presentation is organised around the mechanical effect of the magnetic field. In the first part of the article, a simple coil is studied and the difficulty associated to the realisation of the mechanical model of such assembly is highlighted. In the second part, a ferromagnetic part is added. Its effect on the field distribution and its effect on the mechanical stresses are studied. These two parts, coil and core, are linked by the magnetic field and such a link is important. It appears, for example, that any change on the geometry of the coil is sufficient to change the mechanical behaviour of the magnetic core. In the third part, numerical simulations are applied. It has been possible to understand how the physical phenomena link these different substructures to each other. By using this knowledge, coming from experiments and numerical simulations, a prototype of low-noise inductor was performed and is presented in the last part.

\section{The source of noise in single coils.}

A Lorentz-force acts immediately on a wire when it is plunged into a magnetic field. Even if such a local law is well known, its mechanical effect is not easily described. The behaviour of wires in a coil is different from the behaviour of a single wire, alone and moving free. Using experiments, it appears that, without magnetic core, the coils may produce undesirable noises. Even if it is natural to associate the Lorentz-forces to the sources of such mechanical vibrations, the analysis of the spectrum 
attached to the audible noise shows that this phenomenon is more complex. The calculation of the emitted noise, using the Helmholtz equation, only takes into account the linear behaviour (Bauer et al. 1999). Generally, numerical simulations are used to calculate the distribution of the magnetic induction around the wires and the effect of Lorentz-forces are considered as an effect that pushes all the wires and joins them together (Fig. 1.). Using simulations where each wire is modelled individually, it appears that the effect of these forces on the coil is not homogeneous. It means that such a stress distribution on the coil may excite modes of free vibrations of the coil and also, may introduce unbalanced forces that can generate mechanical deformations (Leohold J. and al. 1988; Tang Y.Q. and al., 1990Wang Z.Q. and al., 2002). A publication has described such deformations through the study of a single coil (Euxibie E. and al., 1986). According to the linear hypothesis, the intensities of the magnetic forces that are acting on the wires are associated to the square of the input current. The frequencies of the noises attached to these mechanical vibrations are twice the frequency of this input current.

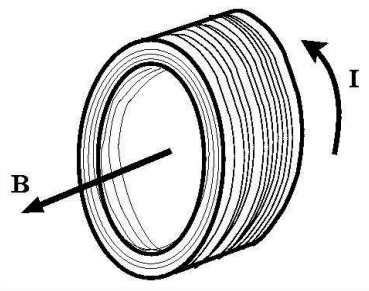

1.a

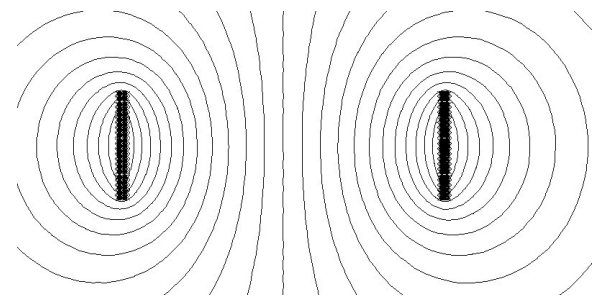

$\mathbf{1 . b}$

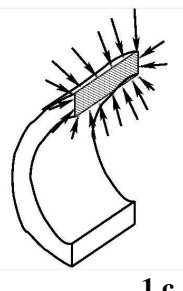

1.c

Fig. 1. With a single coil, (1.a) magnetic flux lines can be calculated (1.b). Under such magnetic field distribution, the magnetic stress joins wires together (1.c), the forces intensities on such wires are not homogeneous and coil deformations appear.

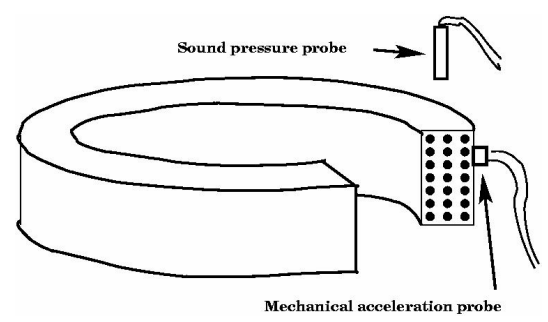

2.a

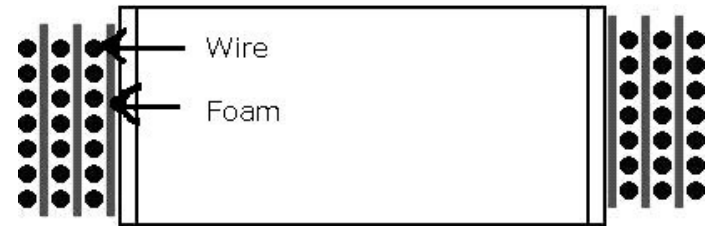

2.b (structure of the test coil)

Fig. 2. A test coil with spaced wires is built (2.a). The emitted noise and the level of vibration are measured. The space distance between the wires is similar to their diameter. A material with a very low elasticity modulus is used to maintain the wires (2.b). This material can damp mechanical vibrations and the wires can move under electrodynamics forces.

Using the energy approach, this mechanical energy, associated to the mechanical vibrations, can be considered as losses. These losses can produce noises and also heat. This heat comes from the mechanical friction of the wires and has no relation with the joule losses. The mechanical effect of Lorentz forces, and especially the noises, can be highlighted with a simple experiment (Fig. 2). A test coil, built with wires that are not tightly maintained, is examined under a sinusoidal current. The level of mechanical vibrations and the level of emitted noises are measured.

The sinusoidal current is imposed in this test coil and in a coil built with a more conventional process. The intensity of the current is constant and its frequency is variable. The mechanical acceleration and the level of sound pressure associated to the noise are measured on these two coils. It appears that the level of mechanical vibration is higher in the test coil (Fig. 3). Unfortunately, this conclusion is too simple. During the experiment associated to the test coil, a free mode of vibration is excited. It is associated to a $600 \mathrm{~Hz}$ frequency sinusoidal current and consequently the frequency of the mechanical effects is $1200 \mathrm{~Hz}$.

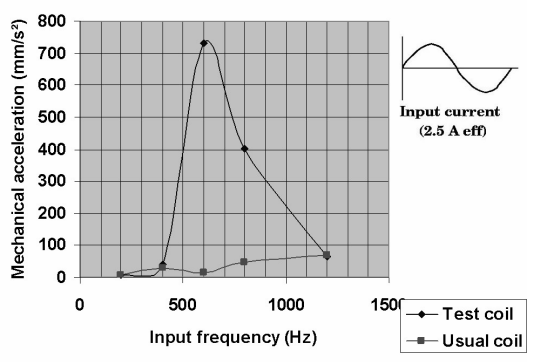

Fig. 3: Measures of intensities of the mechanical accelerations on the coils associated to the first harmonic of the input current. For the test coil, the wires can move and for the conventional coil, the wires bend. A mode of free vibration appears in the test coil (input current $600 \mathrm{~Hz}$, vibration $1200 \mathrm{~Hz}$ ). 
This experiment seems to show that the manufacturing processes giving a high rigidity in winding would also have a positive impact on the reduction of the emitted noises. Nevertheless, any mechanical structure has a mode of free oscillation and the rigid structures have modes of free oscillation with generally high frequencies. Thereafter, another test bench is performed. Two coils with a similar geometry are studied. The first one is built with strongly linked wires. In the second one, the wires are not linked to each other and each layer is separated by a thin plate from foam. Hence, each wire is free to move and effects on the other wires are less important. The physical effect that is expected is the electrodynamics force. When a sinusoidal current is imposed in a wire, this one generates a magnetic induction and this magnetic induction has no global effect on the wire. Such result is only obtained when there is only one wire. If other wires are introduced, the magnetic induction induced electrodynamics forces on each wire. Without links between wires, the behaviour of a single wire can easily be expressed using finite element resolution. When confronted to sinusoidal currents at a frequency of $50 \mathrm{~Hz}$, the frequency of these electrodynamics forces is 100 $\mathrm{Hz}$. The magnitude of such forces can only be calculated with a finite element resolution. Nevertheless, the results associated to these experimental benches are interesting. The mechanical behaviour of the test coil corresponds perfectly to the behaviour predicted by the linear hypothesis. The main frequency of the mechanical strength is twice the input current frequency. In figure 4 , the mechanical behaviour of the coil, containing linked wires, does not always correspond to this hypothesis. It means that modelling coils must be done with caution when mechanical effects are calculated.
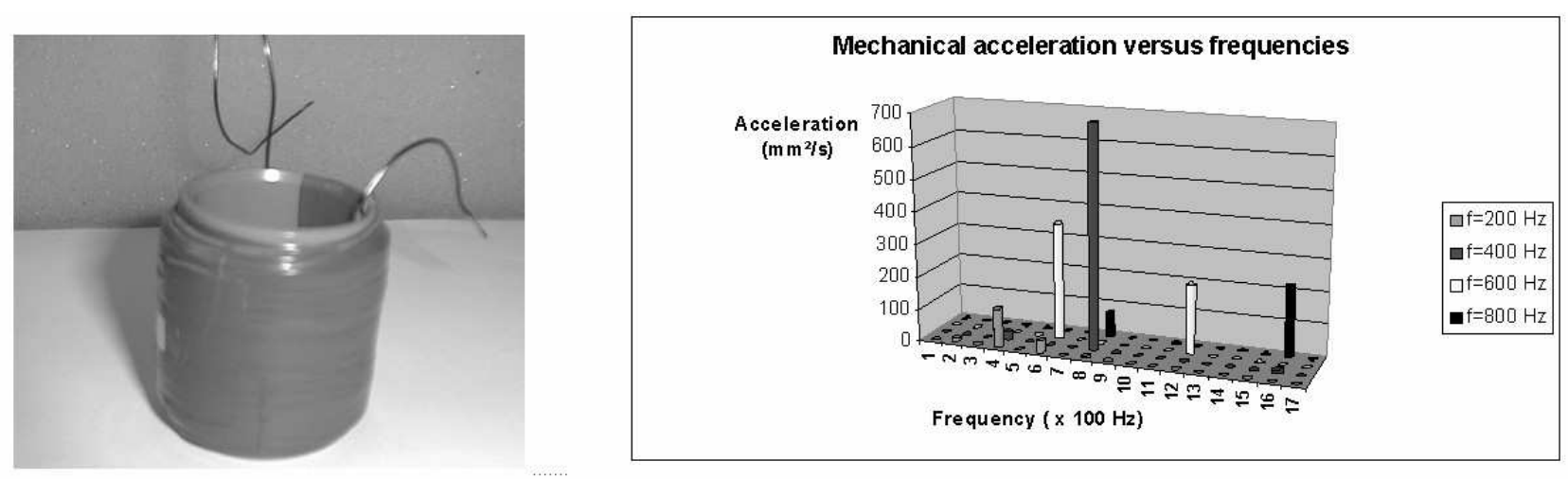

4.a
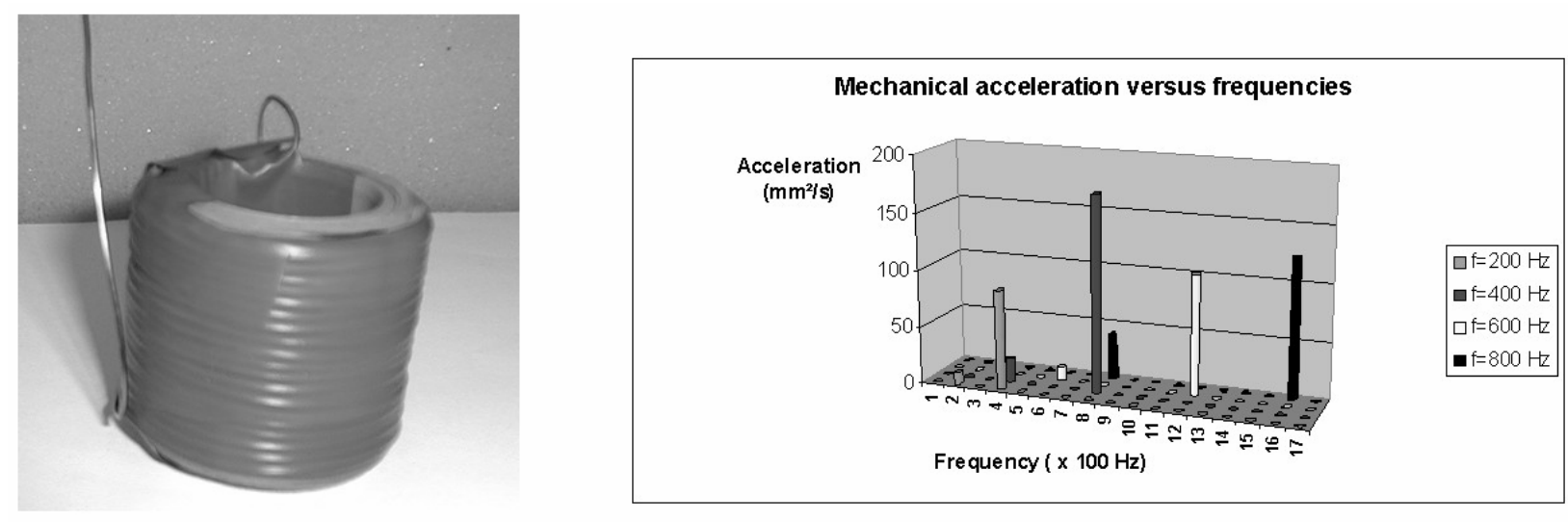

4.b

Fig. 4. Two coils, having similar geometries but different wiring structures are tested. In (4.a), the wires are bent and it appears that the mechanical behaviour does not correspond to the linear behavior. The most important component of the signal associated to the mechanical acceleration is not always associated to the double of the current frequency. In (4.b), the wires are free to move, the mechanical response of the coil correspond to the linear theory, the most important level of vibration is linked to the double of the current frequency. In addition, the level of mechanical vibration is lower in this configuration and no free mode of oscillation is excited. 
An ultimate experiment is performed on the effect of the linked wires. The magnitude of the local mechanical acceleration is related to the current coupled to the magnetic induction. Hence, it means that the magnitude of the mechanical acceleration versus the square of the current must be a straight line under linear behaviour hypotheses. If the experiments do not correspond to this behaviour, such mismatching can be associated to a non linear behaviour (Fig. 5)

\section{Mechanical acceleration versus current magnitude}

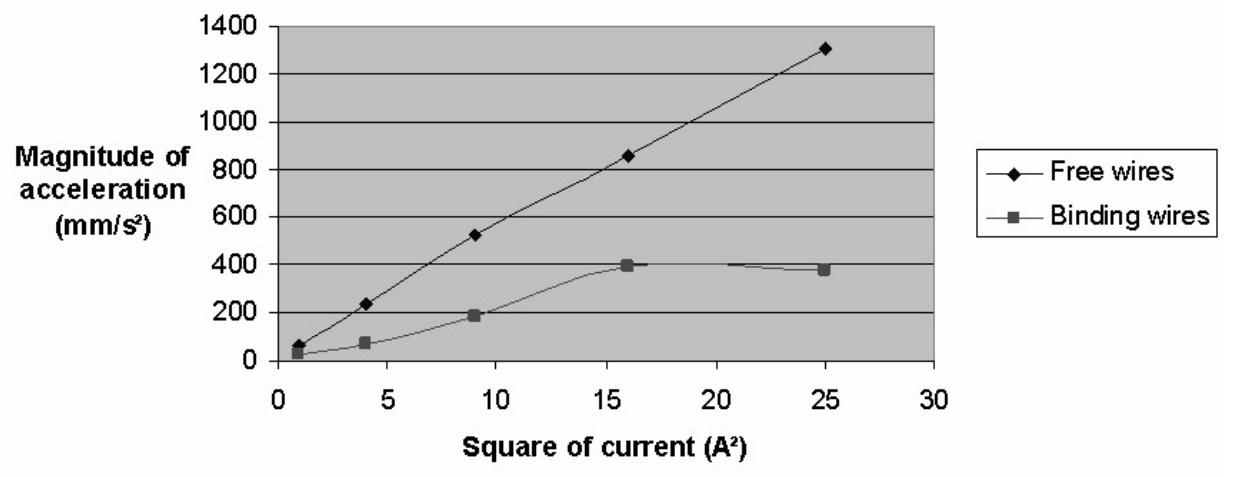

Fig. 5. A sinusoidal current at a fixed frequency, $600 \mathrm{~Hz}$, is imposed in the two preceding coils. The mechanical acceleration is measured for different current intensities. The coil, using free wires, corresponds to the linear behavior theory. It means that the mechanical acceleration is linear with the square of the current intensity.

Linear laws are suitable if quick calculations of the mechanical accelerations need to be performed. The hypotheses, used in such calculations, only point out the behaviour of a single wire. In addition, a non linear behaviour induces an increase in the spectrum of frequencies of the mechanical accelerations. Even if a real linear behaviour can not be obtained, it is possible to minimize the effect of such unexpected behaviours. Now, if the level of sound pressure associated to each mode of vibration is taken into account, it appears that a decrease of the mechanical acceleration is generally linked to a decrease of the sound pressure. Unfortunately, a high level of mechanical acceleration is not always associated to a high level of sound (Fig. 6); it is very difficult to predict the noise level which will be associated to a mechanical vibration applied to a coil. In this first step, it can be concluded that linking wires to each other is not a solution. The effect of this process essentially concerns the modification of the frequencies of the modes of free vibrations. It induces mechanical behaviours that do not correspond to linear hypotheses. Any local positive effect of any solution will disappear if a mode of free vibrations of the coil is excited by the electrodynamics forces.

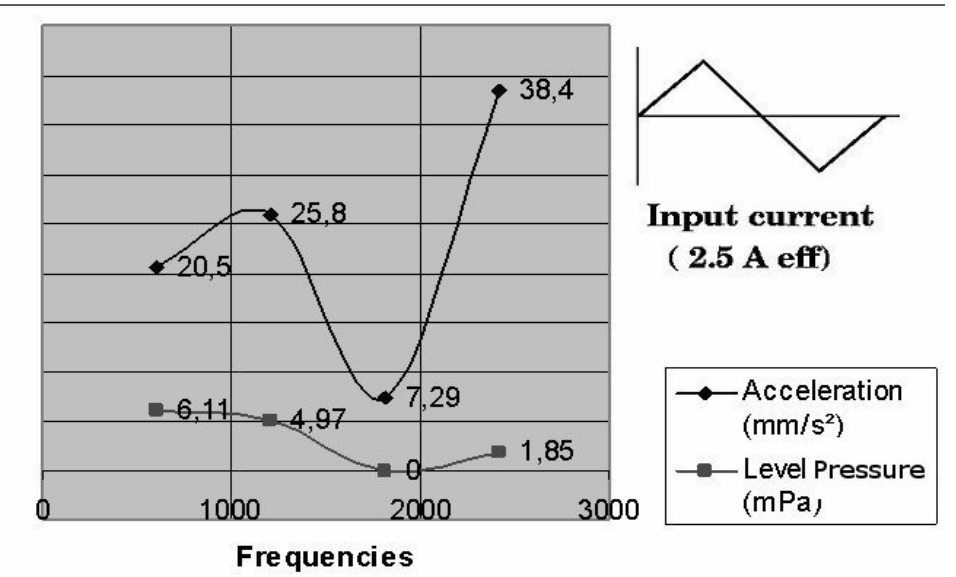

Fig. 6. A current having fixed intensities, a variable frequency and a triangular shape is imposed in a coil. It appears that the level pressure of emitted noise depends on the level of mechanical acceleration. But it could not be concluded that a low level of mechanical acceleration induces a low level of sound pressure. 


\section{Effect of magnetic core on magnetic induction and wires response.}

With a magnetic core, the magnetic induction, especially its distribution on the wires, is modified. Such disturbances are sufficient to change the effect of the electrodynamics forces on the wires. On figure 7, it appears that the wires which are located in the centre of the coil undergo mechanical effects which expel them out of the coil. The introduction of a magnetic core also implies an increase in the level of magnetic induction and also an increase in the mechanical stress on the wires. This one is able to induce winding deformations (Naiqiu S. and al., 2002). Near the wires, without magnetic core, the magnetic flux is under 4,5 $\mathrm{mT}$ and with the magnetic core, the magnetic flux increases to $6,5 \mathrm{mT}$. Such an increase is not sufficient to explain the increase in emitted noise.

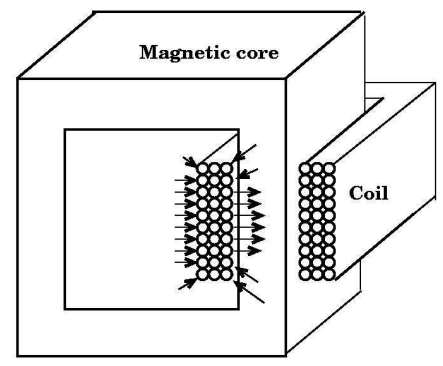

Fig. 7. The magnetic core changes the flux distribution. When the Lorentz-force is calculated on each wire, it appears that all the forces are not directed toward the coil. Some wires, especially the inner wires, are expelled out of the coil.

A basic phenomenon must be pointed out. In this study, the magnetic core can be compared to the magnetic circuit and the membrane of a loud speaker. Magnetic forces generated by the coils are acting on this structure and a noise is emitted. To understand such unconventional phenomena, a simple example is taken into account (Fig. 8).

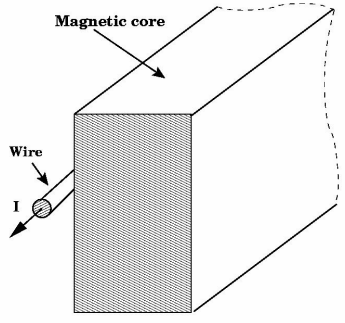

8.a

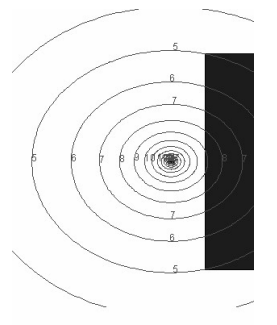

8.b

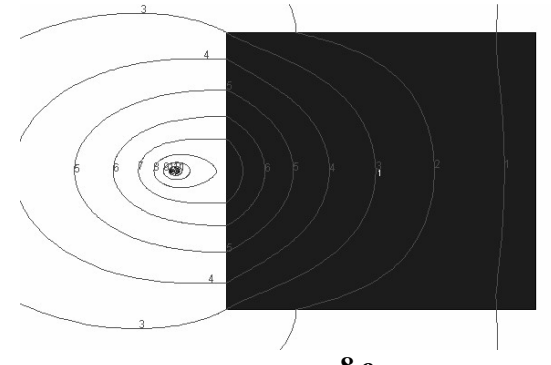

8.c

Fig. 8. A wire, carrying a direct current, is placed near a body (8.a). If this body has no magnetic property, the flux lines are presented in figure $8 . b$, flux lines are circular. If this body has a magnetic property, the flux lines are given in figure 8.c.

A wire is placed near a core (Fig. 8.a). In the first case (Fig. 8.b); the core does not have magnetic properties. In the second case (Fig. 8.c); the core has a high relative magnetic permeability. In the first case, the field distribution presents a perfect symmetry. The flux lines are circular and the centre is the wire. The calculation of the forces acting on such a wire, using the Lorentz formulation, gives a result equal to zero. But in the second case, the proximity of a body, having a high relative permeability, changes the result. The flux lines are bent and the magnetic induction in the centre of the wire is not equal to zero. It means that such a wire undergoes external forces and has to move. The wire is attracted by the body. To calculate the force acting on the wire, the Lorentz formulation is the best solution (1). The body is built with a high relative permeability material and it can be considered that the tangential component of the magnetic induction at its surface is equal to zero. Consequently, it is possible to use a reduced form of the local force formulation provided by the energy principle (2). This equation gives the magnetic pressure that appears on any elementary surface of the magnetic core. Integrating this pressure on each side of the core makes it possible to calculate the force acting on each side.

$$
\begin{aligned}
& \vec{F}=I \cdot d \vec{l} \wedge \vec{B} \\
& \vec{F}=\int_{\text {boundary }} \vec{F}_{S} \cdot d \vec{S}=\int_{\text {boundary }} \frac{1}{2} \frac{1}{\mu_{0}} B_{n}^{2} \cdot \vec{n} \cdot d \vec{S}
\end{aligned}
$$


With a numerical simulation, based on the vector potential formulation, the distribution of magnetic induction can be calculated. The total force acting on the wire can be evaluated with equation (1). The strength of the magnetic induction used to calculate the force acting on the wire is the magnetic induction in the centre of the wire. The total force acting on the body can be obtained by an integration of the local force density given by equation (2) and such integration can be reduced to the nearest border. When a direct current of 7.5 A is imposed in the wire, the magnitude of the total force F1 is equal to $1,0510^{-4} \mathrm{~N} / \mathrm{m}$ and is directed to the body. The magnitude of the total force F2 is equal to $1,03410^{-4} \mathrm{~N} / \mathrm{m}$ and is directed outside (Fig. 9). It immediately appears that such mechanical effects are balanced. In addition, in this example, one wire is set near the side named (ab). If another wire is set near the side named (cd) at the same level, it is possible to balance the total forces acting on the core. In this case, local forces are not equal to zero and it remains mechanical effort. It is sure that such effort does not induce a movement of the magnetic core and will not generate a high level of vibration if variable current is set in the wire.

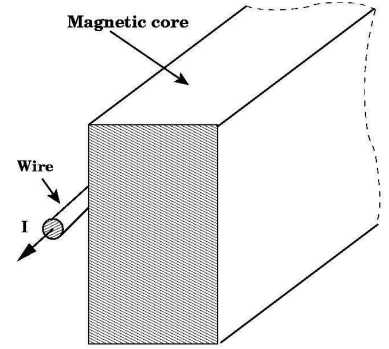

9.a

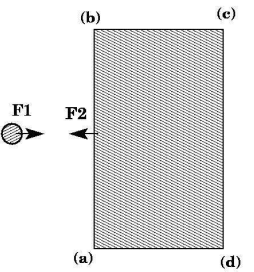

9.b

Fig. 9. The system presented in figure 9.a has to evolve. The wire and the magnetic core are attracted (9.b). F1 and F2 can be calculated using the Lorentz equation and the local force equation given by the energy method.

In the first part of this article, it has been underlined that the determination of the behaviour of a coil is not directly linked to the calculated forces. Non linear behaviours and the heterogeneity of the structure of the coil disturb any calculation. Nevertheless, the calculation of the forces acting on wires is necessary and such result is easily obtained with the Lorentz formulation. The total force acting on a part of a coil is also the total force that is acting on the nearest border of the magnetic body. Knowing this, it will appear that such information is suitable to decrease the level of mechanical vibration of the magnetic core. The structure of the core is rigid and its free modes of vibration may be excited by forces coming from wires. Exciting one mode is enough to increase much more the level of emitted noise. The prediction of the noise level is not easy even if the main physical phenomenon is identified (Colin G.G., 1979; I.E.E.E. Committee report, 1968; Sasaki and al., 1987; van der Veen, 2000). It should be a better solution to suppress the free modes of vibration.

\section{Mechanical behaviour of the magnetic core.}

The mechanical structure of the magnetic core is essentially designed in terms of low electric power losses. The magnetic core is built with silicon-iron magnetic sheets. Even if air-gaps are inserted, the magnetic core remains an assembly of parts that are firmly maintained. Such a solution and especially this mechanical assembly presents drawbacks. Simulations show that the frequencies of the modes of free vibrations associated to this magnetic core parts are audible. With experiments, it appears that the level of these noises, associated to such frequencies, is high enough to overcome any other source of sound (Fig.10.).
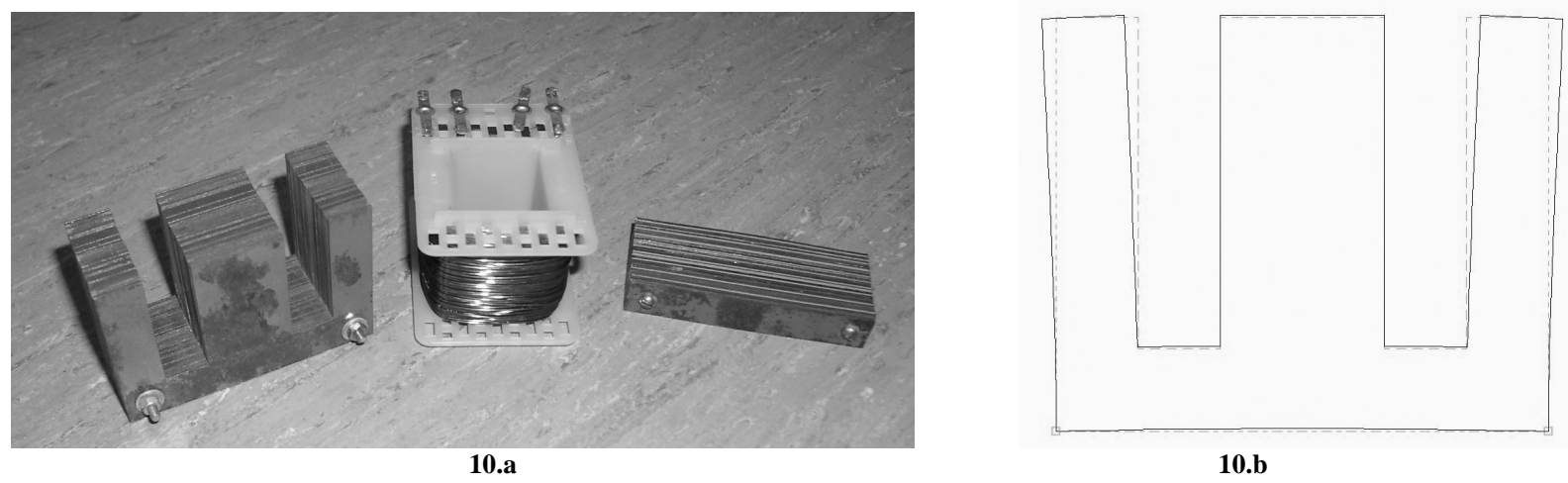

Fig. 10. An inductor is built with iron parts, air gaps and one coil (10.a). The main part of the magnetic core $10 . \mathrm{b}$ is sensible to mechanical excitations and presents an undesirable free mode of vibration. In figure 10.b, the frequency associated to this deformed shape is $2822 \mathrm{~Hz}$. 
The main part of this core presents a mode of free oscillation the frequency of which is $2822 \mathrm{~Hz}$. When a sinusoidal current, the frequency of which is half this value, is imposed in the coil, a high level of vibration is observed. Such an undesirable behaviour will occur whenever the spectrum of the current contains a component at this frequency. Knowing that any frequency and any shape of current are possible in an inductor; a better solution in order to decrease sound level should be to suppress the free modes of vibration associated to the magnetic core. The main idea consists in suppressing the magnetic core rigidity. All the parts are geometrically similar and the air gaps are distributed along the structure and placed at all the corner. These air gaps are filled with a material which damps out the vibrations. In addition, the parts can also move freely (Fig 11).
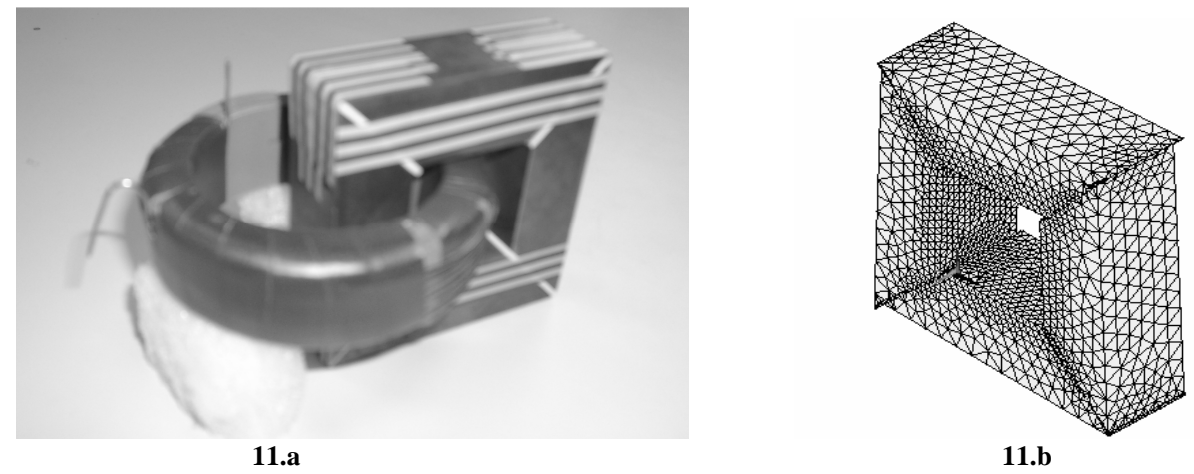

Fig. 11. This inductor is built with iron parts and air gaps are distributed along the magnetic core (11.a). The air gaps are filled with a material which is able to damp vibrations, the free modes of oscillation attached to this material have low frequencies and no deformation is noticed on the rigid parts (11.b).

The mechanical behaviour of the test inductor and especially the modes of free oscillations are studied with numerical simulations that take only into account the densities and the elasticity of the materials used. Two different ranges of frequencies appear. The first one starts at $73 \mathrm{~Hz}$ and stops at $400 \mathrm{~Hz}$. The second one starts at $8000 \mathrm{~Hz}$. The first range of frequencies depends on the properties of the material inserted in the air gap and also depends on the method used to join each part to the others. The second range depends on the shape of each elementary magnetic part. It means that it is possible to build a structure without modes of free oscillation in a large range of frequencies. It is known that active solutions can reduce the level of mechanical vibration; this study, using a passive solution, also provide the same result (Mandayam S and al., 1994; Teoh C. and al., 1998).

\section{Prototype of a low-noise industrial inductor.}

The tests performed in the two preceding parts use sinusoidal currents. This current shape enhances the mechanical effect of the magnetic field. It means that magnetic effects induced by such currents in inductors are less important than the effect induced by a pure sinusoidal current having the same efficiency value. Such currents are not usual in industrial inductors. The real currents generally associate a direct current and a less important modulated current. The frequencies associated to the modulated current are linked to the switch frequencies of the power supply. The effects of magnetostriction in these devices are naturally undervalued. Such a conclusion is not present for motors and transformers (Vandevelde L. and al. 2003). The first concept of a new magnetic structure is studied; each elementary part of this structure has free modes of oscillations whose frequencies are high. Two different solutions have been studied and both magnetic and mechanical behaviours were examined. For each elementary part, the lowest frequency of mode of free vibration has been calculated. It is always above $4000 \mathrm{~Hz}$. As shown in figure 12, the geometry of the structure remains conventional. The typical inductance value for this low-noise inductor is similar to the typical value of an industrial inductor, $100 \mathrm{mH}$ at $50 \mathrm{~Hz}$.

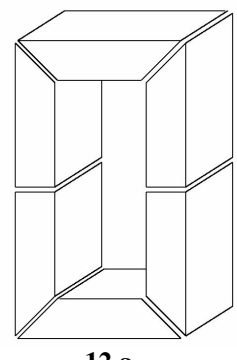

12.a

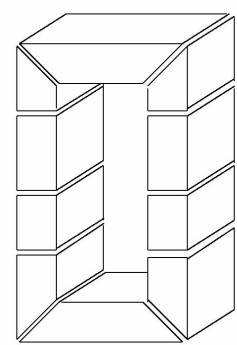

12.b

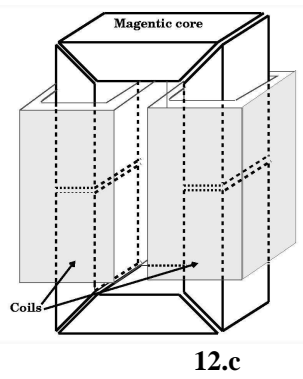

12.c

Fig. 12. The prototype of the low-noise inductor is built with iron parts and air gaps are distributed along the magnetic core (12.a and 12.b). Two coils are added and simulations are used to calculate the typical value of inductance and also the effect of the wires on the core (12.c). 
An undesirable phenomenon must be pointed out. With such a structure, it is possible to define a range of frequencies where each elementary part of the prototype presents a neutral behaviour for mechanical external excitations. It must be noticed that for the whole structure, a free mode remains and it must be dealt with. In particular, the coils are able to induce mechanical strains on the structure (Kumar and $a l ., 2005$ ). These strains can excite this remaining free mode. Even if the material used to fill the air gaps is able to damp vibrations, it will not suppress this mechanical phenomenon (Fig. 13).
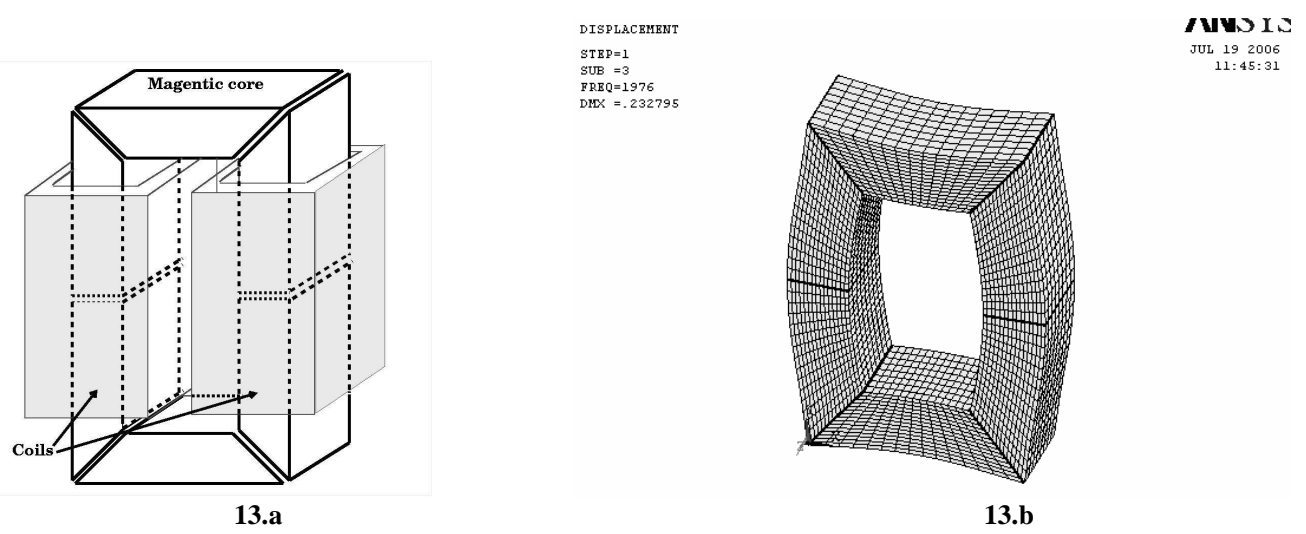

Fig. 13. Even if the rigidity of the magnetic core disappears (13.a), an undesirable mode of free vibration remains (13.b). Its associated frequency is in an audible range $(1976 \mathrm{~Hz})$.

The second part of the article shows that the magnetic core and wires are attracted. It is more reliable to calculate this force on the wires. The magnetic field distribution on the core is not reliable, especially near corners, and consequently this computation is a problem (Barré and al. 2005). Doing this, it is assumed that the global force acting on one side of the core is also the global force acting on the coil. Moreover, regarding the vertical part of the core, the symmetry of this structure indicates that forces densities should be equivalent on the upper and the lower part of the core. It is not necessary to calculate the forces acting on the core; these forces are balanced with the forces that are acting on each part of the coil. Hence, through numerical simulations, it is possible to point out the suitable areas for each part of the coils. In these areas, the forces that are acting on each part of the wires are balanced. Consequently, the forces that are acting on the magnetic core are also balanced. This solution diminishes the mechanical excitation on the magnetic core. (Fig 14.). The main effect of this solution is linked to the mode of free oscillation shown in figure 13.b and its amplitude can drastically be diminished.

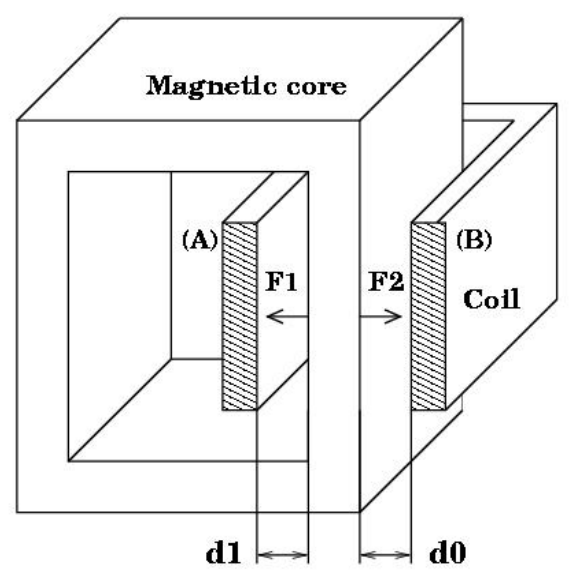

Fig. 14. With a suitable position for each part (A and B) of the coil, F1 is equal to F2 and the calculated free mode of vibrations (1976 Hz) will not be excited. In this test inductor, $\mathrm{d} 1=2 \mathrm{~mm}$ and $\mathrm{d} 0=44 \mathrm{~mm}$.

The coil is divided into two different substructures (Fig. 14). The first one (A) is surrounded by the magnetic core and the second one $(\mathrm{B})$ is outside the magnetic core. All the positions for these two substructures are not possible. In particular, the first part of the coils (A) must be very close to the magnetic circuit. The second part (B) must be placed at an average distance from the core. As shown in figure 15, this choice increases the force associated to the wires. It also gives a more suitable homogeneity to the direction of each elementary force associated to each wire. About the prototype, the distance $\mathrm{d} 0$ is $44 \mathrm{~mm}$ and the distance $\mathrm{d} 1$ is $2 \mathrm{~mm}$. Generally such effects of Lorentz-forces are studied in coils under short circuit conditions (Wang Z.Q. and al., 2002). 

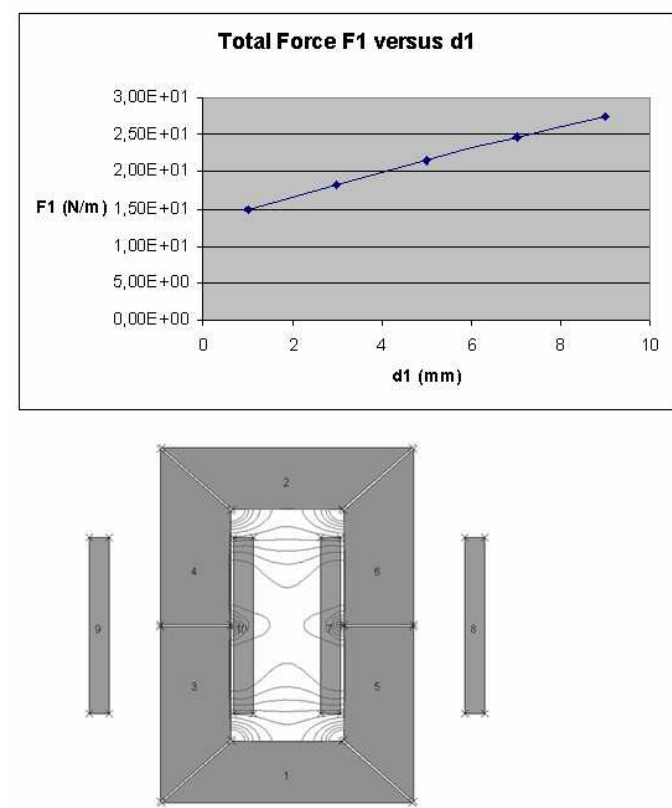
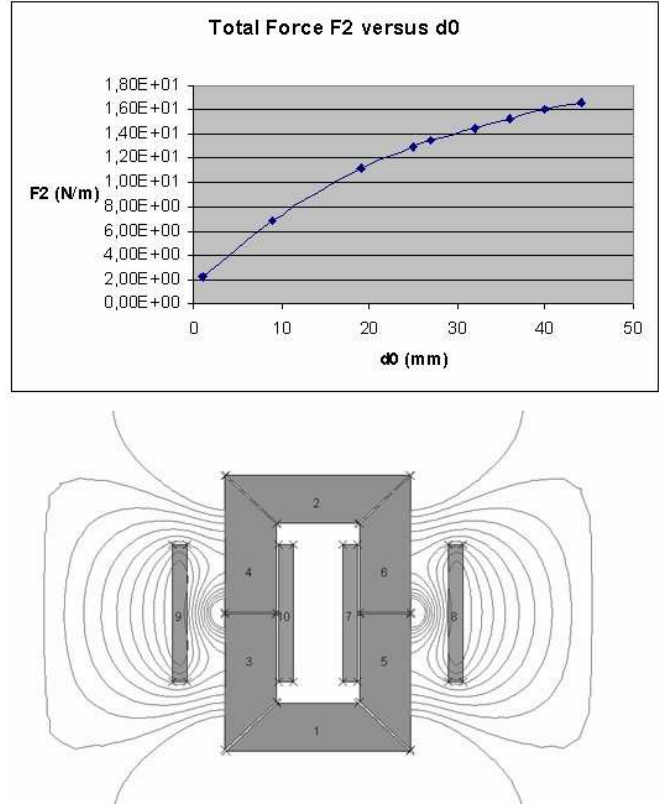

15.b

Fig. 15. Calculation of the forces acting on each side of the magnetic core: F1 and F2 are obtained calculating the force acting on the each substructures of the coil. F1 is calculated using the magnetic flux distribution in the inner area (15.a) of the inductor and F2 is calculated using the magnetic flux distribution outside of the magnetic core (15.b).

The most undesirable phenomena are the mechanical vibrations with low frequencies. Due to air gaps, the strength of the flux remains below the saturation level of the magnetic material used. It explains why the simulations can be achieved with a linear model. Simulations use the magnetic vector potential formulation (3). About the mechanical aspect, especially the free modes of oscillation, the basic equation is the equation (4) where, $[\mathrm{K}]$ is the rigid matrix of the structure, $[\mathrm{M}]$ is the mass matrix, [U] the displacement vector associated to the whole structure at $\omega$ the pulsation of the free mode of oscillation (Zienkiewicz and al. 1989).

$$
\begin{aligned}
& \operatorname{Curl}\left(\frac{1}{\mu} \operatorname{Curl}(\vec{A})\right)=\vec{J} \\
& {[K][U]=\omega^{2}[M \rrbracket[U]}
\end{aligned}
$$

\section{Comparison between the prototype inductor and the industrial inductor.}

Considering the prototype inductor, the measure of the typical inductance value at $50 \mathrm{~Hz}$ is $89 \mathrm{mH}$. The mechanical behaviour is not acceptable for low frequencies. The rigidity of the structure, due to the material used to damp the vibration, is low and high levels of vibration can be produced. Nevertheless, the associated level of emitted noise is low and these low frequencies are not considered as disturbances for the human ear. To compare the prototype inductor and the industrial inductor, a load, a pure resistor, is coupled to an electronic power supply and the two inductors are added to this circuit. The main frequency of the electronic power supply can be modified; the level of sound pressure and the mechanical vibrations are thereafter measured. The prototype inductor can minimise the level of emitted noise for average frequencies only. Above 800 $\mathrm{HZ}$ and under $9000 \mathrm{~Hz}$, the prototype has a flat behaviour. The main aspect that must be pointed out is the disappearance of any mode of free vibration in this range of frequencies. The industrial inductor presents noisy modes in this range and the sound pressure immediately reaches a high level. It appears that the switching frequency of the power supply does not change the behaviour of the core; the modes of free oscillations appear at each frequency (Fig. 16). 

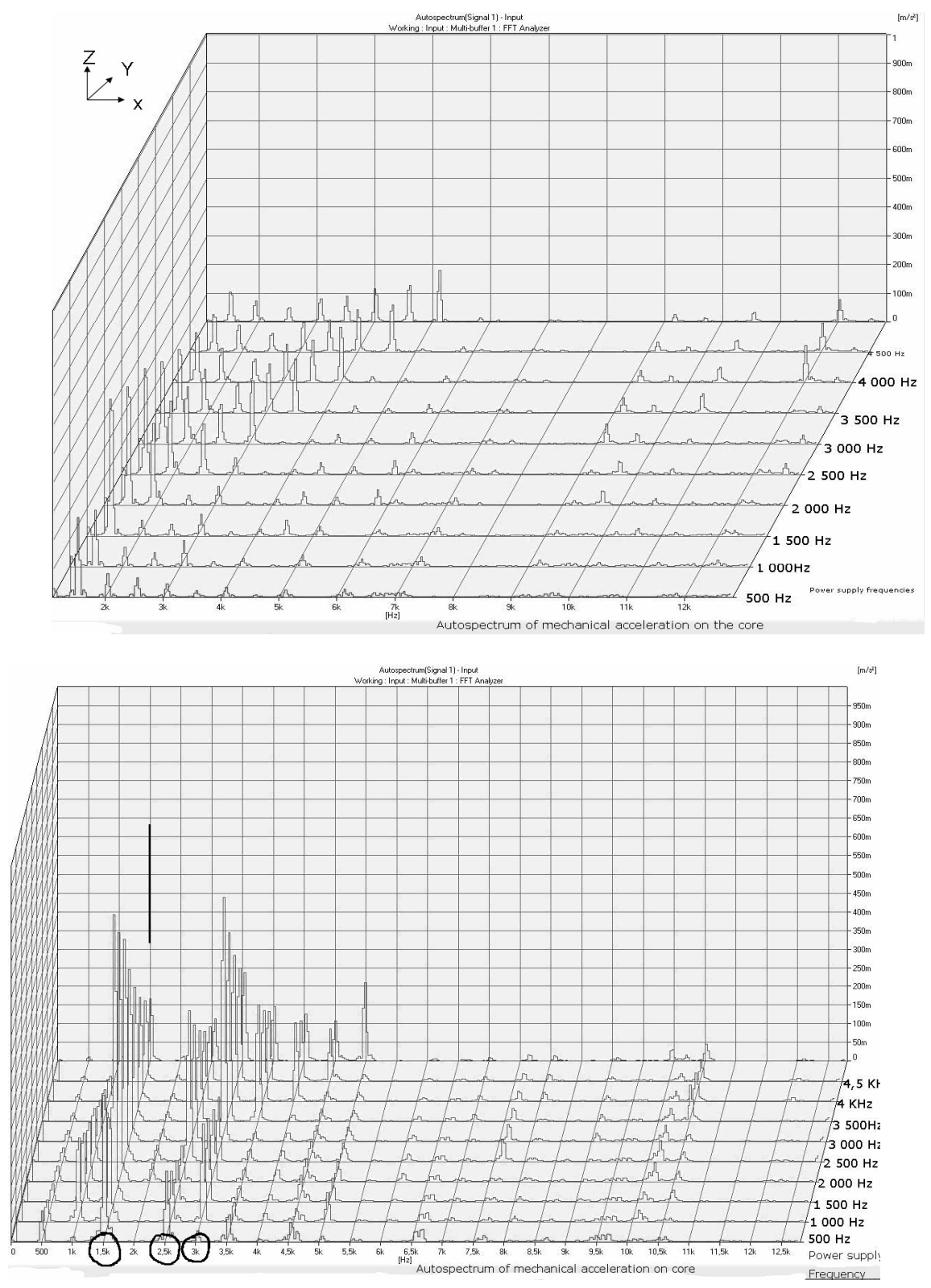

Fig. 16. The industrial inductor has many free modes of oscillation. For example, The $\mathrm{Z}$ reference holds the strength of the mechanical acceleration measured on the prototype or industrial inductor. It appears in figure $16 . \mathrm{b}$ that free modes of oscillation exist on the industrial inductor especially at 1,5;2,5;3,0 Khz (X reference). A high level of acceleration is noticed at these frequencies for all the switch frequencies used in the power supply ( 500 to $4500 \mathrm{~Hz}, \mathrm{Y}$ reference). The prototype of the inductor (16.a) does not present modes of free oscillation in the range of frequency usually used by the power supply.

\section{Conclusion.}

The Inductors are commonly used in the power supplies containing electronic switches. Even if the mechanical structures of these inductors are similar to the mechanical structures of transformers, the air gaps drastically modify their physical behaviors. In the first part of this article, wires carrying currents and surrounded by magnetic fields induced an audible effect. But even if the Laplace force exists, it is not the main effect associated to the emitted noise in the industrial inductors. In the following part, using experiments, it appears that the magnetic core is important sources for such noises. Such result is explained by the presence of air gaps distributed along the magnetic circuit. They induce magnetic discontinuities and consequently generate sources of mechanical efforts that can excite free modes of oscillation of the whole structure. In keeping air gaps in the structure, a possible solution to decrease the emitted noise consists in handling the frequency of the free modes of oscillation. In the last part of this article, this solution is experimented with a prototype inductor. Wires are not the main source of noise, but they are always a source of mechanical stress. In particular, putting the core in the middle of the coil is not suitable. This geometrical symmetry does not imply a balance of stress on each side of the core. Cyclic efforts remain on the core. They can be diminished with a suitable position of the coils in the core. Hence, it appears that the recommended position is not the 
solution used in industrial inductors. As a conclusion, this study introduces a design of a new core structure which presents a neutral behavior in front of any mechanical excitation. It means that a reduction of noise is possible with a passive solution if this one is integrated in the first step of the inductor design.

\section{References}

Barré O. and Brochet P., "Geometrical singularities effects on the calculation of the magnetic forces studied by an equivalent behaviour approach", COMPEL, The International Journal for Computation and Mathematics in Electrical and Electronic Engineering,Vol. 26, No. 1, February 2007, pp. 49-62.

Bauer T. and Henneberger G., Three-dimensional calculation and optimization of the acoustic field of an induction furnace caused by electromagnetic forces. I.E.E.E. Transcations on magnetics, Vol. 35, N 3, May 1999, pp 1598-1601.

Colin G. G., A method for predicting the audible noise emissions from large outdoors power transformers, I.E.E.E. Transaction on power apparatus, Vol. PAS-98, N³, May/June 1979, pp 1109-1112.

Euxibie E., Coulomb J.-L., Meunier G. and Sabonnadière J.-C., Mechanical deformation of a conductor under electromagnetic stresses », I.E.E.E. Transaction on magnetics, Vol. 22, N5, September 1986, pp 828-830.

I.E.E.E Committee Report, Bibliography on transformer noise, I.E.E.E. Transaction on power apparatus and systems, Vol. Pas$87, \mathrm{~N}^{\circ} 2$, February 1968, pp 372-387.

Kumar S., Kumbhar G. B., Kulkarni S. V., Aiyar R.P.R.C. and Desai S.V., Electromagnetic Forming: A case study of coupled magneto-mechanical formulation, In proceeding of ISEF 2005- XII International Symposium on electromagnetic fields, in Baiona, Spain, 15-17 September 2005.

Leohold J. and Silvester P.P., Calculation of transformer winding resonances by a specialized finite element method employing substructures, I.E.E.E. transaction on power system, Vol. 3, № 3, August 1988, pp 1385-1389.

Mandayam S., Udpa L., Udpa S.S. and Sun Y.S., A fast iterative finite element model for electrodynamics and magnetostrictive vibration absorbers, I.E.E.E. Transaction on Magnetics, Vol. 30, № 5, September 1994. pp 3300-3303.

Naiqiu S., Can Z., Fang H., Qisheng L. and Lingwei Z., Study on ultrasonic measurement device for transformer winding deformation. In Proceedings of PowerCon 2002. International Conference on Power System Technology, 13-17 October 2002, Kunming, China, Vol. 3, pp 1401-1404.

Sasaki T., Takada S., Ishibashi F., Suzuki I., Noda S. and Imamura M., Magnetostrictive vibration of electrical steel sheets under a non sinusoidal magnetizing conditions, I.E.E.E. Transaction on magnetics, Vol. Mag-23, $\mathrm{N}^{\circ} 5$, September 1987, pp 3077-3079.

Teoh C., Soh K., Zhou R., Tien D. and Chan V., Active noise control of transformer noise, Proceedings of EMPD '98. International Conference on Energy Management and Power Delivery, Singapore, 3-5 March 1998.

Tang Y.Q. and Qiao J.Q., Numerical calculation of short circuit electromagnetic forces on the transformer winding, I.E.E.E. Transaction on magnetics, Vol. 26., $\mathrm{N}^{\circ}$ 2, March 1990.

van der Veen M., Measuring acoustic noise emitted by power transformers, AES $109^{\text {th }}$ Convention, Los Angeles, U.S.A., 22-25 September 2000 .

Vandevelde L. and Melkebeek A.A., Magnetic forces and magnetostriction in electrical machines and transformer cores, I.E.E.E. Transaction on magnetics, Vol. 39, N³, May 2003, pp 1618-1621.

Wang Z. Q., Wang M. and Ge Y.Q., The axial vibration of transformer winding under short circuit condition, In Proceedings of PowerCon 2002. International Conference on Power System Technology, 13-17 October 2002, Kunming, China, Vol. 3 , pp 1630-1634.

Zienkiewicz O.C. and Taylor R.L., "The finite element method" $4^{\text {th }}$ edition, McGraw-Hill, 1989. 